\title{
Modelling X-ray Pulse Profiles of Millisecond Pulsars
}

\author{
D. A. Leahy ${ }^{1}$, S. Morsink ${ }^{2}$ and W. Tian ${ }^{3}$ \\ ${ }^{1}$ Dept. of Physics and Astronomy, University of Calgary, \\ Calgary, Canada T2N 1N4 \\ email: leahy@ucalgary.ca \\ ${ }^{2}$ Dept. of Physics, University of Alberta, \\ Edmonton, Canada \\ email: morsink@ualberta.ca \\ ${ }^{3}$ National Astronomical Observatories, CAS, \\ Beijing 100012, China \\ email: tww@bao.ac.cn
}

\begin{abstract}
The modelling of X-ray pulse profiles from accreting millisecond pulsars is a way to infer masses and radii of neutron stars. We briefly describe how a pulse shape encodes information on the mass and radius, but also depends on other parameters such as hot spot location and observer viewing angle. A numerical model that we have developed is then described. The model includes light bending, time-delay effects, and Doppler effects for photons. The model accounts for oblateness of the neutron star, caused by the rapid rotation, and for scattered light from the surface of the accretion disk. The millisecond pulsar SAX J1808-3658 has multiple observations taken during different outbursts. The observed pulse shapes vary greatly, and it is a challenging test to fit the different observations. Some of the latest results are given.
\end{abstract}

Keywords. dense matter, equation of state, gravitational lensing, stars: neutron

The modelling of X-ray pulse profiles from accreting millisecond pulsars, is one method used to infer masses and radii of neutron stars, although it has not reached the accuracy obtained for the pulse profile model of Hercules X-1 (Leahy, 2004). For a summary of methods see the review paper by Lattimer \& Prakash (2007) Gravity plays a strong role in the propagation of light from the neutron star to observer, as well as on the structure of the neutron star itself. Thus light-bending must be included in any model of observations of neutron stars. Because of the rapid rotation of millisecond (ms) pulsars, Doppler effects (boosting and aberration), which depend on emitter velocity, affect the observed X-ray pulse shapes. As a consequence of light bending and Doppler effects, pulse shapes carry useful information on the mass, radius and surface shape of the neutron star.

The numerical model that we have developed includes light bending and time-delay effects, as well as Doppler effects for photons. This model is described in detail in a series of papers (listed below). The model also accounts for oblateness of the neutron star, caused by the rapid rotation. and includes a scattered light component from the surface of the accretion disk.

The calculation of pulse shapes is done by ray tracing, using the geodesic equations of general relativity (GR). Full calculations use the numerical GR metrics for rotating neutron stars. Expansion of the geodesic equations in the metric for a rotating neutron star yields redshift and Doppler factors Cadeau et al. (2007). Time-delays are important for pulse shapes, but the difference between Schwarzchild and Kerr metrics are not important Cadeau et al. (2005): both have similar (but small) differences from the correct neutron star metric. Omitting the fact that rapid rotation causes the neutron star to 
have an oblate shape produces large errors in the pulse shapes (for spin frequencies $<\sim$ $300 \mathrm{~Hz}$; Cadeau et al. 2007). An approximation method to the full GR calculation was presented by Morsink et al. (2007). This was essential as a practical method calculating pulse shapes because the full GR calculation is too compute-intensive to be used in pulse shape fitting programs. This approximate method is called the oblate-Schwarzschild approximation. It was applied to SAX J1808-3658 by Leahy et al. (2008).

Including data from two or more different observations of the same millisecond X-ray pulsar is very useful to distinguish fixed parameters (mass, radius and observer viewing angle) from variable parameters (hot spot location size and emission properties). Joint fits of more than one pulse shape was first done using data on the millisecond X-ray pulsar XTE J1814-338 by Leahy et al. (2009). It was then extended to observed pulse profiles of SAX J1808-3658 by Morsink \& Leahy (2011). This work also introduced a scattered light component, for X-rays scattered from the neutron star hot spot off the accretion disk. This was needed in order to provide satisfactory models for the observed pulse shapes. Leahy et al. (2011) applied the oblate Schwarzchild model with scattered light with a joint fit to pulse profiles from 3 epochs from XTE J1807-294.

The millisecond pulsar SAX J1808-3658 has multiple observations taken during different outbursts as described by Hartman et al. (2008). It is a challenging test to fit the different observations, in large part because of the large number of free parameters needed to describe the time-variable emitting region, the neutron star and observer geometry. Details will be presented in a future publication.

Figure 1 compares model fits with and without scattered light. The $3-4 \mathrm{keV}$ band has a significant contribution from a blackbody spectral component with isotropic phase function; the 9-20 keV band is dominated by the power-law spectral component with an electron scattering phase function. The model with disk-scattered light is a significantly better fit to the observed pulse profile. Figure 2 shows the best fit values of mass and radius for different pairs of 2-epoch fits, and the 3 sigma allowed region for the 2-epoch fit using 1998b4 and 2002b3 data sets. We have carried out 3-epoch fits which yield

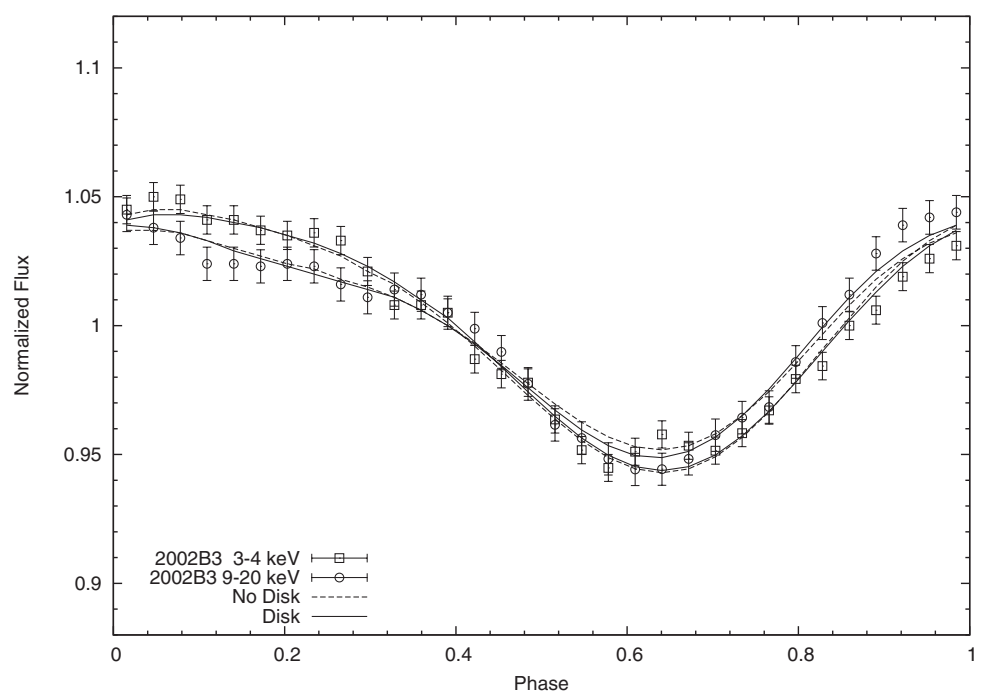

Figure 1. Pulse profiles from SAX J1808-3658 for 2002b3 epoch in two energy bands (open symbols with error bars), and the best fit model pulse shape fits for model without scattered light from the accretion disk (dashed line labelled "No Disk") and for model with scattered light (solid line labelled "Disk"). 


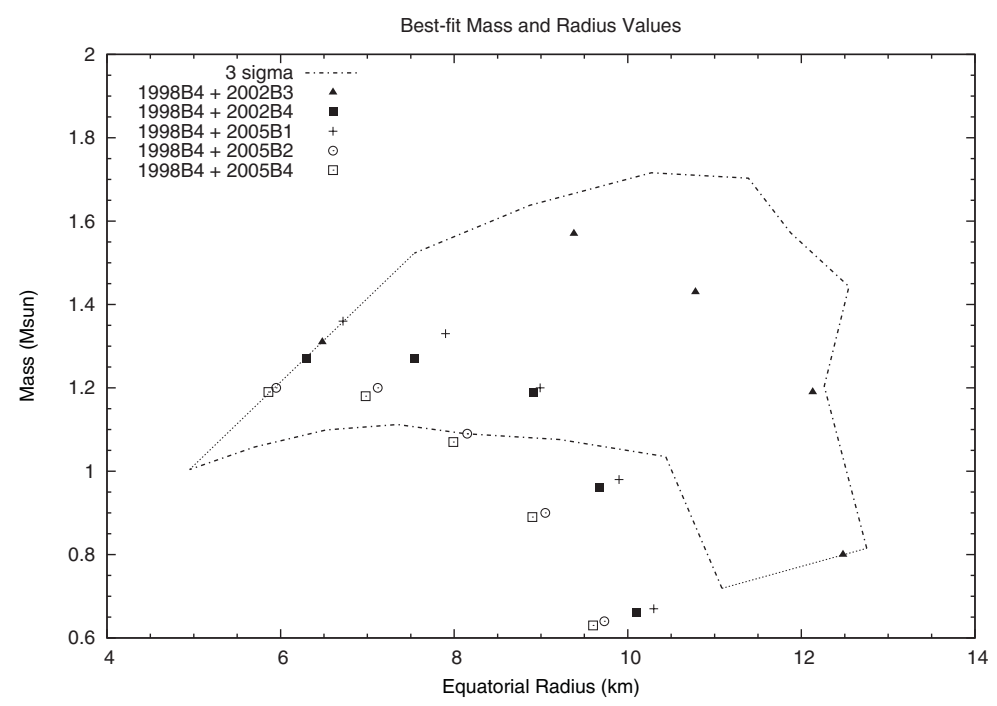

Figure 2. Best fit values of $\mathrm{M}$ and $\mathrm{R}$ for the labelled pairs of pulse profiles from SAX J1808-3658 (solid points) plus the 3-sigma limits on $\mathrm{M}$ and $\mathrm{R}$ for the 1998b4- 2002b3 joint pulse shape fit.

consistent best fit mass and radius and also a significantly smaller 3 sigma allowed region. Next is planned improvements in the fitting algorithm and then results from joint 4-epoch fits.

\section{References}

Leahy, D. A. 2004, ApJ, 613, 517

Lattimer, J. M. \& Prakash, M. 2007, Physics Reports, 442, 109

Cadeau, C., Leahy, D. A., \& Morsink, S. M. 2005, ApJ, 618, 451

Cadeau, C., Morsink, S. M., Leahy, D., \& Campbell, S. S. 2007, ApJ, 654, 458

Morsink, S. M., Leahy, D. A., Cadeau, C., \& Braga, J. 2007, ApJ, 663, 1244

Leahy, D. A., Morsink, S. M., \& Cadeau, C. 2008, ApJ, 672, 1119

Leahy, D. A., Morsink, S. M., Chung, Y.-Y., \& Chou, Y. 2009, ApJ, 691, 1235

Morsink, S. M. \& Leahy, D. A. 2011, ApJ, 726, 56

Leahy, D. A., Morsink, S. M., \& Chou, Y. 2011, ApJ, 742, 17

Hartman, J. M., Patruno, A., Chakrabarty, D., et al. 2008, ApJ, 675, 1468 